\section{ANALYSIS OF IDO-1 EXPRESSION ON DENDRITIC CELLS AND FACTORS INFLUENCING ITS UP- AND DOWNREGULATION IN PANCREATIC CANCER}

Michael Hollingsworth, Kamiya Mehla, Kirsten Eberle, Ying Huang, Aleata Triplett, Paul Grandgenett, Clara Mundry*, Thomas Caffrey. University of Nebraska Medical Center, Omaha, NE, United States

Background Pancreatic Ductal Adenocarcinoma (PDAC) is bad. An immunosuppressive tumor microenvironment (TME) with an excess of immunosuppressive immune cells and cytokine/ chemokine factors contribute to local and systemic immunosuppression in PDAC. ${ }^{1}$ Our laboratory has generated singlecell RNA-Sequencing (scRNA-Seq.) data from spleens derived from PDAC patients and healthy counterparts. This data demonstrates the existence of dendritic cell (DC) subsets with a tolerogenic phenotype. These DCs display increased expression of several markers, including Indoleamine 2,3-dioxygenases (IDO-1 and IDO-2), widely accepted as markers for a specific population of DCs: tolerogenic DCs. These cells evoke an immunosuppressive signal leading to activation of regulatory $\mathrm{T}$ cells and MDSCs as well as apoptosis of CD8+ and CD4+ effector T cells. ${ }^{2}{ }^{3}$

Methods To validate our scRNA-Seq. data, we performed pilot investigations harvesting DCs from the spleen of PDAC patients and healthy subjects. Besides examining human specimens, we also investigated the IDO-1 expression on splenic DCs from tumor-bearing mice, orthotopically implanted with LSL-KrasG12D; LSL-Trp53R172H/+; Pdx1-Cre (KPC)-derived cell lines. It is known that tumor-derived exosomes can impact DC-differentiation to a tolerogenic phenotype. ${ }^{4}$ Exosome purification using differential ultracentrifugation is a well-established method in our lab and optimized for our autopsy samples. We analyzed tumor-derived exosomes for their potential in modulating IDO-1 expression on DCs in in vitro assays. Briefly, we incubated DCs with different exosome concentrations and harvested the cells for RNA-sequencing and flow cytometry.
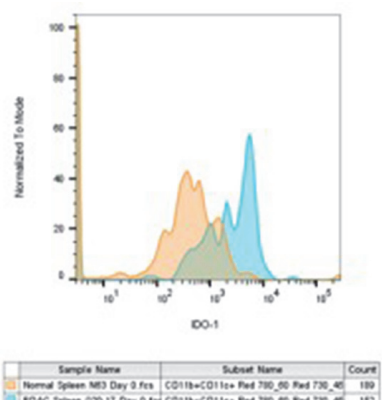

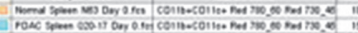

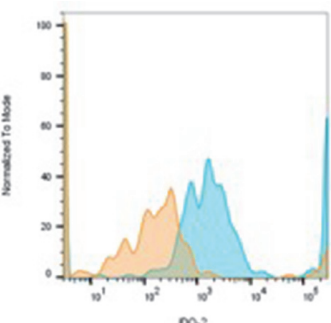

$\infty$

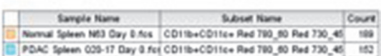

Abstract 654 Figure 1 Expression of IDO-1 and IDO-2 on DCs from PDAC spleen (blue) .and normal spleen (orange)

Results Compared to normal spleens, DCs from PDAC spleens displayed higher expression of IDO-1 (figure 1). Additionally, KPC-tumor-bearing mice showed higher expression of IDO-1 on DCs from the spleen and blood compared to wild-type mice. Further investigating the influence of PDAC-derived exosomes on marker expression on DCs have shown an apparent increase in expression of IDO-1 when culturing splenic-derived DCs with tumor-derived exosomes (figure 2).

Conclusions While tolerogenic DCs are essential in regulating the homeostasis between immune response and immune tolerance, ${ }^{5}$ several studies have shown IDO-1 overexpression in cancer. Investigating tolerogenic DCs is an essential part of our lab's efforts to understand the nature of the immune response in PDAC. Future directions for this project include determining molecular pathways that regulate the expression of IDO-1. Additionally, we will investigate downstream mechanisms through which exosomes modulate the switch to a tolerogenic phenotype. We also plan to further characterize different splenic DC populations by evaluating their interplay with other immune cells in the context of antigen-specificity and other factors influencing these cells' properties.

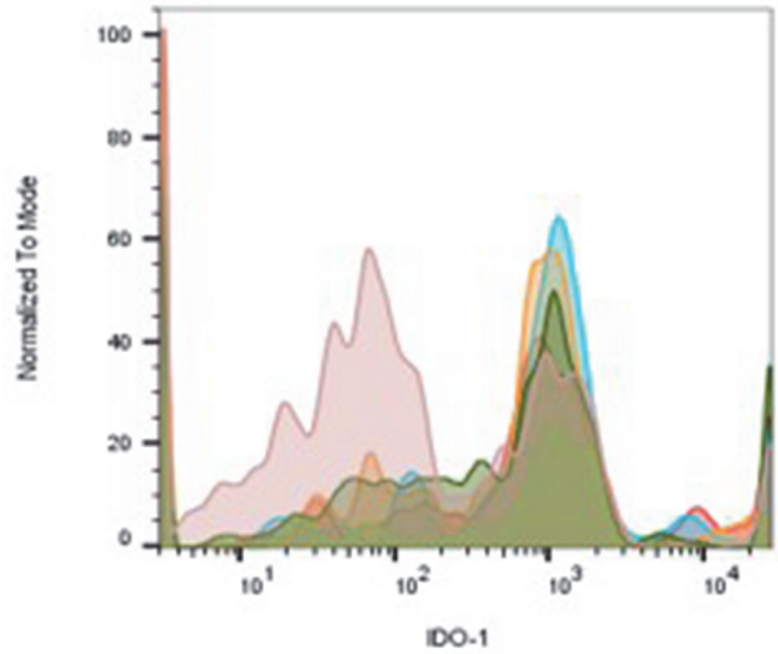

\begin{tabular}{|c|c|c|c|}
\hline & Sample Nlame & Subset Name & Count \\
\hline D & Nes Day 0 DCs.fes & CD11b+CDilet & 800 \\
\hline$\square$ & DCs 24 h 100 ugmt.fes & CDIIbeCDilse & 180 \\
\hline 口 & DCs 24 h 50 ugmd.fes & CDitb+CDilet & 62.0 \\
\hline D & DCs $24 \mathrm{~h} 20$ ugw.fos & CDilbeCDites & 120 \\
\hline$\square$ & DCs $24 \mathrm{~h} 10 \mathrm{ugml}$ fos & CDIfbecDilet & 147 \\
\hline 口 & DCs 24 h $5 \mathrm{coml}$.fes & CDilb+CDilet & 144 \\
\hline
\end{tabular}

Abstract 654 Figure 2 Change in expression of ID0-1 through treatment of DCs with different concentrations of tumor-derived exosomes

\section{REFERENCES}

1. Mundry CS, Eberle KC, Singh PK, Hollingsworth MA, Mehla K. Local and systemic immunosuppression in pancreatic cancer: targeting the stalwarts in tumor's arsenal. BBA - Reviews on Cancer 2020;1874(1):188387

2. Liu M, Wang X, Wang L, Ma X, Gong Z, Zhang S, Li Y. Targeting the ID01 pathway in cancer: from bench to bedside. Journal of Hematology \& Oncology 2018;11(1):100

3. Hornyák L, Dobos N, Koncz G, Karányi Z, Páll D, Szabó Z, Halmos G, Székvölgyi $\mathrm{L}$. The role of Indoleamine-2,3-Dioxygenase in cancer development, diagnostics, and therapy. Frontiers in immunology 2018;9:1.

4. Bronte V, Pittet MJ. The spleen in local and systemic regulation of immunity. Immunity 2013;39(5):806-818.

5. Domogalla MP, Rostan PV, Raker VK, Steinbrink K. Tolerance through education: how tolerogenic dendritic cells shape immunity. Frontiers in Immunology 2017:8:1764.

Ethics Approval This study was approved by the University of Nebraska Medical Center Ethics Board; approval numbers IRB\#: 440-16-EP and IRB\#: 091-01.

http://dx.doi.org/10.1136/jitc-2021-SITC2021.654 